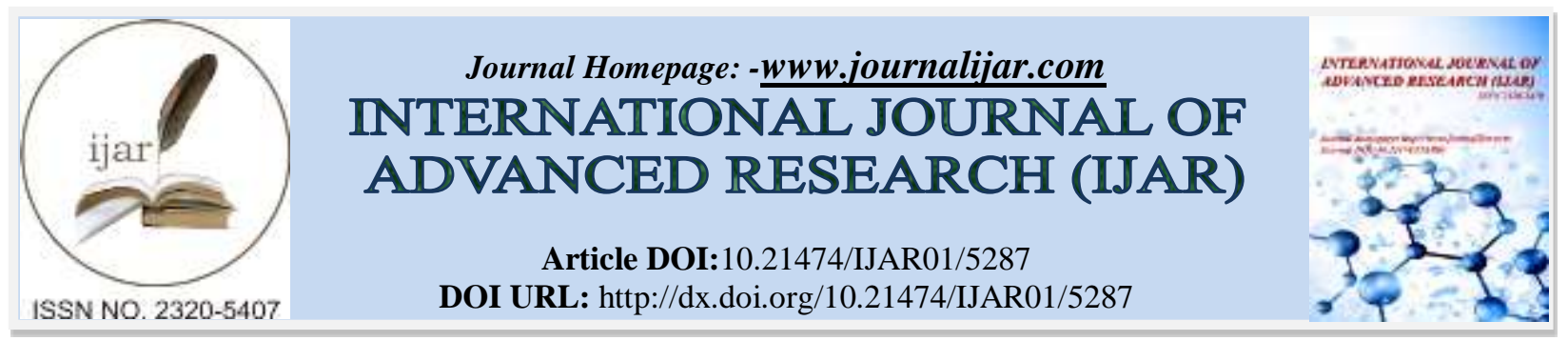

RESEARCH ARTICLE

\title{
STUDY OF NUCLEATED RBCS IN CORD BLOOD OF NEONATES WITH MECONIUM STAINED AMNIOTIC FLUID.
}

Seema Puri, Krishna Jindal, Vijay Suri and Gaurav Puri.

\section{Manuscript Info}

Manuscript History

Received: 26 June 2017

Final Accepted: 28 July 2017

Published: August 2017

Keywords: -

Nucleated red blood cells, meconium stained amniotic fluid, Cord Blood.

\begin{abstract}
Background: The purpose of this study was to investigate variations in Nucleated Red Blood Cells in cord blood associated with meconium stained liquor in newborn babies.

Method: Ninety six babies were registered for the study with forty eight as cases and forty eight as controls. Cord blood was collected and number of Nucleated Red Blood Cells determined. It was prospective case control study.

Result: Babies delivered with meconium stained amniotic fluid exhibited a significantly higher number of NRBCs/100 WBC (11.29 \pm 4.77) than control group (3.13 \pm 1.67$)$. Absolute NRBC count was statistically highly significant in case group.

Conclusion: This simple laboratory test that can be performed in a resource limited setting is also cost effective and is highly specific tool in predicting perinatal asphyxia due to meconium staining of liquor.

Hence NRBC count can be a useful part of armamentarium for evaluation of perinatal asphyxia.
\end{abstract}

Copy Right, IJAR, 2017,. All rights reserved.

\section{Introduction:-}

Meconium is a mucoviscidic odorless sterile substance and is the first stool passed by newborn.

Meconium stained amniotic fluid is composed of swallowed amniotic fluid debris, bile pigments, intestinal secretions and desquamation of gastrointestinal tract of fetus. Amniotic Fluid surrounds the fetus in intrauterine life providing low resistance space suitable for growth and development. ${ }^{(1)}$.

Infants born with MSAF are at increased risk of hypoxia. Perinatal asphyxia remains a significant cause of perinatal morbidity and mortality ${ }^{(2)}$.

The parameters used for predicting or defining asphyxia include Apgar score, acidemia of the umbilical cord artery, fetal heart monitoring, patterns of heartbeat, $\mathrm{pH}$ metry of the blood of fetal head, increased level of serum erythropoietin, and presence of meconium in amniotic fluid ${ }^{(3)}$

However, to date no single marker of perinatal asphyxia has shown good predictive efficacy and only a combination of various indices can help in an early diagnosis of perinatal asphyxia. Recently, the count of umbilical cord NRBC for every 100 white blood cells has been introduced as a marker of perinatal asphyxia ${ }^{(4)}$. 
The mechanism of increase in NRBCs in hypoxia is attributed to increase level of cord blood erythropoietin within 1 to 4 hours following acute hypoxia; promoting release of NRBCs from bone marrow stores as high titers of erythropoietin accelerates mitotic division of normoblast from bone marrow ${ }^{(5)}$.

It is a simple test that can be done at rural areas even in primary health centre, which are backbone of healthcare in India. This study aims to find relationship between MSAF and NRBC counts.

\section{Materials and Methods-}

The present observational study was conducted in Department of Pathology, Adesh Institute of Medical Sciences and Research, Bathinda over a period of one and a half year from 1-04-2014 to 30-09-2015.

Study included 96 newborns and among them 48 were cases which had neonates born with MSAF. The control group included neonates born with clear amniotic fluid.

Detailed history of mother was taken and those with diabetes, hypertension, preeclampsia, COPD and H/O smoking during pregnancy were excluded from the study.

Details of labor and delivery were recorded. After rupture of membranes amniotic fluid was examined for presence and absence of meconium. The babies born with MSAF were labeled as cases and those with clear amniotic fluid as controls.

From all newborns cord blood was taken aseptic conditions $2 \mathrm{ml}$ of cord blood was collected immediately after clamping and cutting the umbilical cord.

Sample was taken in EDTA coated vaccutainer and was processed in automated cell counter. Blood smears were prepared and examined to evaluate complete hematological profile.

NRBCs were counted against 100 WBCs. Absolute NRBC count was calculated using following formula:

Absolute NRBC count $(/$ cumm $)=$ WBC count $x$ NRBCs /100 WBC

\section{Results-}

Ninety six newborn babies were enrolled for the study. Mean maternal age, gestational age, parity, mode of delivery, sex and birth weight of infants (Mean \pm SD) is presented in Table no. 1.

Table no.1-Comparison of demographic profile of case and control group.

\begin{tabular}{|l|l|l|l|}
\hline Parameters & Case group & Control group & p value \\
\hline Maternal age (yrs) & $25.15 \pm 4.32$ & $24.33 \pm 2.80$ & 0.121 \\
\hline Gestational age (wks) & $38.52 \pm 1.34$ & $37.98 \pm 1.12$ & 0.034 \\
\hline Male/Female & $25 / 23$ & $26 / 22$ & 0.807 \\
\hline Parity & $1.9 \pm 0.8$ & $1.8 \pm 0.6$ & 0.768 \\
\hline Birth weight (gms) & $2554.27 \pm 565.57$ & $2856.25 \pm 403.79$ & 0.003 \\
\hline Mode of delivery(ND/CS) & $27 / 21$ & $31 / 17$ & 0.157 \\
\hline
\end{tabular}

From the table no. 1 it can be seen that newborns of case group had higher gestational age

as comparison to control group. Birth weight was significantly lower in case group as compared to control group. The most common mode of delivery was normal vaginal delivery both in cases and controls. Both the case and control groups were comparable in terms of maternal age, sex of infant, parity and mode of delivery. There was no significant difference of these parameters in both case and control group.

NRBC/100 WBC levels were estimated at birth in umbilical cord sample and results were compared between the meconium stained and control group. Mean NRBCs/100 WBC in case and control group were $11.29 \pm 4.77$ and $3.13 \pm 1.67$ respectively (Table 2). A statistically significant difference was seen on basis of absolute NRBC count (p value $=0.0001)$. The mean absolute NRBC's in cases and control group was $1737.17 \pm 1389.23 /$ cumm and $390.94 \pm$ 259.77/cumm. Number of NRBC/100 WBC in control group ranged from 0 to $10.81 .25 \%$ of babies had NRBCs less than 5/100 WBCs and $18.75 \%$ babies had NRBCs in range of 5-10/100 WBCs. In the case group the NRBCs 
ranged from 3 to $24.4 .17 \%$ of newborns had NRBCs less than 5/100 WBCs, $45.83 \%$ had NRBCs in range of 5-10, $33.33 \%$ had NRBCs in range of $11-15,10.42 \%$ had 16-20 NRBCs and $6.25 \%$ of babies had 21-25 NRBCs/100 WBC.

The NRBCs/100 WBC level was significantly higher in the cases group as compared to the control group with a $\mathrm{p}$ value of 0.0001 .

Table no 2:- Comparison Of Hematological Profile in Control And Case Groups.

\begin{tabular}{|l|l|l|l|}
\hline $\begin{array}{l}\text { Hematological } \\
\text { characteristics }\end{array}$ & Case & Control & p value \\
\hline Hemoglobin (gm/dl) & $14.84 \pm 2.18$ & $14.51 \pm 1.70$ & 0.418 \\
\hline WBC Count ( /cumm) & $13564.17 \pm 4856.53$ & $12096.04 \pm 3364.14$ & 0.089 \\
\hline Platelet count (/ Lac) & $2.7 \pm 0.9$ & $2.9 \pm 0.8$ & 0.151 \\
\hline NRBCs/100 WBCs & $11.29 \pm 4.77$ & $3.13 \pm 1.67$ & 0.0001 \\
\hline $\begin{array}{l}\text { Absolute NRBCs } \\
(\text { /cumm) }\end{array}$ & $1737.17 \pm 1389.23$ & $390.94 \pm 259.77$ & 0.0001 \\
\hline
\end{tabular}

Table no. 2 shows the hematological characteristics of study population. In the present study mean hemoglobin is $14.84 \pm 2.18 \mathrm{gm} / \mathrm{dl}$ in case group and $14.51 \pm 1.70 \mathrm{gm} / \mathrm{dl}$ in the control group. No significant difference was found on basis of hemoglobin concentration $(\mathrm{p}$ value $=0.418$ ). The mean WBC Count was $13564.17 \pm 4856.53 / \mathrm{cumm}$ and $12096.04 \pm 3364.14 /$ cumm in the case and control group respectively. In differential count predominant population is that of polymorphs in both groups .No significant difference was found according to platelet count between case and control group.

\section{Discussion:-}

Asphyxia remains one of the main causes of later disability in term infants. The hematopoietic response to hypoxia in utero, the elevated NRBCs /100 WBC count is being hailed as the marker for perinatal asphyxia. Our present study showed that as hypothesized infants with meconium stained amniotic fluid had higher nucleated RBC counts than infants with clear amniotic fluid.

In our present study mean gestational age of $38.52 \pm 1.34$ and $37.98 \pm 1.12$ weeks in case and control group was seen. It was significantly higher in our case group ( $p$ value=0.03). Study by Darkhaneh et al ${ }^{(6)}$ also had similar findings and showed that gestational age of mothers in the control group was significantly lower than the case group . However a study by Boskabadi et al ${ }^{(7)}$ showed that there was no statistically significant difference between the two groups regarding gestational age.

In study conducted by us birth weight was lower in case group than control group. This finding was supported by study conducted by Tungalag et al ${ }^{(8)}$ and Colaco et al ${ }^{(4)}$; they also had lower birth weight in case group. Bayati et al (7) had birth weight of 3055 and 3270 gms in case and control group respectively. They also agreed with our finding as they had lower birth weight in case group.

Mean Hemoglobin concentration was not significant between case and control group. This finding was supported by Darkhaneh et al ${ }^{(6)}$. However Brucknerova et al ${ }^{(3)}$ reported lower hemoglobin values in newborns in case group $(\mathrm{p}$ value $=0.01)$.

Difference in total leukocyte count in both case and control group was not significant in our present study. Our findings were supported by studies conducted by both Darkhaneh et al ${ }^{(6)}$ and Brucknerova et al ${ }^{(3)}$. However Colaco et $\mathrm{al}^{(4)}$ and Boskabadi et al ${ }^{(7)}$ also showed higher WBC count in case group than control group.

In our study babies with meconium stained amniotic fluid had significantly higher cord blood NRBC counts than in babies with clear amniotic fluid. In our case group mean NRBCs/100 WBC and absolute NRBC Count of 11.29 \pm 4.77 and $1737.17 \pm 389.23 /$ cumm was found. In control group mean NRBC/100 WBC and absolute NRBC count of $3.13 \pm 1.67$ and $390.94 \pm 259.77$ was seen. Our difference of NRBCs/100 WBC between case and control was 8.16 units. 
Study by Tungalag et al ${ }^{(8)}$ showed that NRBCs $/ 100 \mathrm{WBC}$ was $11.35 \pm 10.23$ and $4.83 \pm 3.01$ in case and control group respectively. Absolute NRBC/cumm in case and control group was $1652.8 \pm 236.18$ and $746 \pm 62.0$ respectively. They also agreed with our findings as they found mean NRBC Count was significantly higher in babies with MSAF than clear liquor.

Bayati et al ${ }^{(5)}$ reported mean NRBCs/100 WBC Count of $8.2 \pm 1.9$ and 5.6 \pm 2.6 in case and C. Control group respectively. In comparison between case and control group it had been found that mean NRBC difference was 3.6 units and was significantly higher in case than control group $(p$ value $=0.0002)$.

A study conducted by Darkhaneh et $\mathrm{al}^{(6)}$ showed increase in both NRBC/100 WBC and absolute NRBC in newborns with meconium stained liquor than with clear liquor. Colaco et $\mathrm{al}^{(4)}$ also supported our study as their NRBCs were also higher in cases than controls. The mean NRBCs/100 WBC in case and control group were 17.43 \pm 19.86 and $2.97 \pm 4.79$ respectively. This was statistically highly significant ( $\mathrm{p}$ value $<0.0001$ ).

A study done by Phelan et al ${ }^{(9)}$ found the mean NRBCs/100 WBC of $3.4 \pm 3.0$ in non asphyxiated (Control group) and $34.5 \pm 68.3$ in asphyxiated newborns (Case group). This value was higher than reported in our study. However both studies had significant difference between case and control group as in our study.

Study by Abhishek et al ${ }^{(10)}$, Geetika et al ${ }^{(11)}$ and Boskabadi et al ${ }^{(7)}$ also had similar results like our study. Elevation of NRBC in hypoxic situation is based on the fact that any hypoxic event induces a fetal compensatory response in the form of exaggerated erythropoiesis, resulting in influx of immature red blood cells into fetal circulation, levels of which may be correlated with the presence of perinatal asphyxia. To conclude, estimating the number of nucleated RBC per $100 \mathrm{WBC}$ in umbilical cord sample of newborns is a simple test, the sample being obtained non-invasively from an otherwise discarded specimen and analyzed by personnel on equipment readily available in most hospital laboratories.

\section{References:-}

1. Shashikala A, Raghavan S. Perinatal Outcome in relation to mode of delivery in MSAF. Indian J of Pediatrics 1995; 62:63-67.

2. Alam F, Aziz M, Ali M, Hakim S.Evaluation of hematological profile of cord blood and placental histopathology in neonates with perinatal asphyxia. CurrPediatr Res 2012; 16 (2):105-110.

3. Brucknerova I, Ujhazy E, Dubovicky M, Mach M. Early assessment of the severity of asphyxia in term newborns using parameters of blood count. InterdiscToxicol 2008; 1:211-213.

4. Colaco M, Ahmed M, Bajpai R. Study of nucleated red blood cell counts in asphyxiated newborns and the fetal outcome. Int J ClinPediatr2014; 3:79-85.

5. Bayati M, Abid M, Wahbi A. Role of nucleated red blood cells in umbilical cord blood as a marker of neonatal asphyxia with meconium stained amniotic fluid. The Iraqi post graduate medical journal 2014; 13(2):336-341.

6. Darkhaneh RF, Asgharnia M, Yousefi TZ. Comparison of NRBC in term neonatal umbilical cord blood between neonates with meconium stained amniotic fluid and clear amniotic fluid. J Turkish-German Gynecol Assoc 2008;9(2):29-31.

7. Boskabadi H, Maamouri G, Sadeghian $\mathrm{H}$ et al. Early diagnosis of perinatal asphyxia by nucleated red blood cell count. Archives of Iranian medicine 2010;13:275-281.

8. Tungalag L, Gerelma G. Nucleated Red Blood Cell Counts in Asphyxiated Newborns. Open Science Journal of Clinical Medicine 2014; 2(1):33-38.

9. Phelan JP, Ahn MO, Korst LM et al. Nucleated red blood cells-A marker for fetal asphyxia. Am J ObstetGynecol1995; 173: 1380-4.

10. Abhishek MG ,Sarvesh BM. Study of association between perinatal asphyxia and nucleated red blood cell count in umbilical cord blood. International journal of advances in case reports 2015;2:1003-1006.

11. Geetika, Shweta Sikarwar, Gupta Sunita. The Correlation of Clinical Perinatal Asphyxia with Counts of Nrbc/100 Wbc in Cord Blood. Webmed Central Obstetrics and Gynecology. 2011; 2(1):1-9. 